\title{
A simple approach for ultrasound-guided pararadicular injections in the sacral spine: a pilot computer tomography controlled cadaver study
}

\author{
Michaela Plaikner ${ }^{1}$, Hannes Gruber ${ }^{1}$, Christoph Schwabl ${ }^{1}$, Erich Brenner ${ }^{2}$, Reto Bale ${ }^{1}$, \\ Elisabeth Skalla ${ }^{1}$, Alexander Loizides ${ }^{1}$
}

${ }^{1}$ Department of Radiology, ${ }^{2}$ Department of Anatomy, Histology und Embryology, Medical University of Innsbruck, Innsbruck, Austria

\begin{abstract}
Aims: Injection therapies play an increasingly decisive role in the treatment of lower back pain. Cumulative studies could show the benefits of ultrasound-guided instillation procedures in the cervical and lumbar spine. We conducted this study to provide a new simple sonographic approach for pararadicular injections of the sacral spinal nerves and to prove the feasibility and accuracy by means of CT and anatomic dissection. Material and methods: Eight ultrasound-guided injections at four different levels of the sacral spine on a human ethanol-glycerol-embalmed cadaver (S1-S4) were performed. By means of sonography the sacral foramina were identified and the spinal needles were advanced in "in-plane technique" to the medial margin of the respective sacral foramen. Subsequently a solution of blue dye and contrast agent were injected. Then CT scans and anatomic dissection of the cadaver were performed to verify the correct placement of the needle tips and to visualize the dispersion of the injected solution in the respective compartment. Results: Altogether a $100 \%$ success rate for a correct injection could be achieved. CT examination confirmed the correct placement of every needle tip within the intended compartment. Also, the anatomic dissections affirmed the appropriate needle positioning. Moreover, the blue dye dispersion was seen in the correct compartments and around the targeted spinal nerves. Conclusions: Although this study was only performed on cadaveric models, this new sonographic approach for pararadicular injections in the sacral spine allows an easy, precise and unerring needle placement within the dorsal sacral foramen.
\end{abstract}

Keywords: ultrasonography; computer tomography; anatomy; injections; sacrum

\section{Introduction}

In clinical practice lower back pain represents one of the most common musculoskeletal complaints [1]. Even though the underlying reason for the resulting symptoms can be very diverse, the often resulting nerve root com-

Received 05.12.2018 Accepted 18.01.2019

Med Ultrason

2019, Vol. 21, No 2, 125-130

Corresponding author: PD Dr. Hannes Gruber, MD

Medical University of Innsbruck,

Department of Radiology

Anichstrasse 35, 6020 Innsbruck, Austria

E-mail: hannes.gruber@i-med.ac.at

Phone: +43(0)512 50480919

Fax: $+43(0) 51250422758$ pression induces a pain syndrome named lumbosacral radiculopathy [2]. Although exact epidemiologic data are difficult to establish, a prevalence of approximately 3 to $5 \%$ with equal gender distribution has been described [3].

The first sacral nerve (S1) is, due to its position at the lumbo-sacral transition zone, at increased risk of injury (S1 radiculopathy) [4]. At this level a nerve compression leads to pain which spreads along the posterior thigh to the lateral border of the foot and the little toe as well as the sole. Based on the segmental muscle innervation the ankle plantar flexion and the hip extensors are affected. The ankle jerk (Achilles-tendon) reflex is often diminished.

The application of corticosteroid and local anesthetics directly on the compressed nerve has been a repeatedly postulated diagnostic and therapeutic option [5]. There- 
fore, injection therapies are nowadays, beside physical therapy and other rehabilitative methods [1], indispensable and an important alternative to the more invasive and if possible avoidable surgical procedures in the treatment of lower back pain [6].

Different methods are currently available for injections at the lumbosacral region. Although blind access, meaning without any imaging support, has been successfully performed in the last decade, and based on the fact that even in experienced hands an inaccurate needle placement occurs in up to $30 \%$ of cases [7], spinal injections should be obsolete when imaging is available [8]. Nowadays, spinal injections are preferentially performed under fluoroscopic or computer tomographic (CT) guidance: especially CT can be described as the actual "gold standard" imaging tool, based on the anatomic precision and visualization of the needle placement with high accuracy [9]. However, being an incremental widely available, fast, safe and of course ionising-free method enabling real-time needle visualization, ultrasound (US) -guided injection techniques have gained recently wide attention [10] as an alternative modality to the above mentioned techniques. Different approaches have already been described for the cervical [11] and lumbar spine [12], proving the feasibility and effectiveness of US-guided injections in the spine. However, concerning the sacral spine, only a few reports can be found in the literature [13-16].

The sacrum is a roughly triangular bone that counts among the immobile parts of the spinal column. It usually consists of five sacral vertebrae that over life merge to one big bone. At this point lumbosacral transitional vertebrae must be mentioned. They are common congenital anomalies where either the fifth lumbar vertebra is

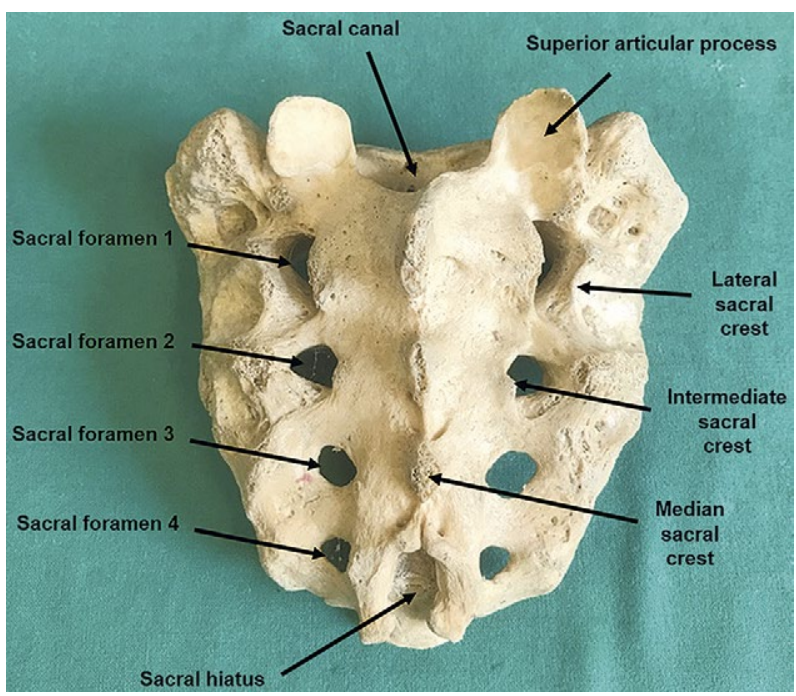

Fig 1. Anatomy of the dorsal aspect of the sacral bone assimilated to the sacrum (sacralisation) or the first sacral vertebra may show transition to a lumbar configuration (lumbarisation) [17]. In the general population the reported prevalence is $4-21 \%$ and their clinical significance with association to low back pain is controversially discussed [18]. The sacral bone serves as an anatomical link between the spinal column and the pelvis and protects the caudal spinal nerves of the spinal cord. From a dorsal view important structures are the median, intermediate and lateral sacral crests (fig 1).

We conducted this pilot study to assess the feasibility of US-guided pararadicular injections in the sacral spine and to evaluate the preliminary accuracy by means of CT and anatomical dissections.

\section{Material and methods}

The study was conducted on a human cadaver, which was preserved using an arterial injection of an alcoholglycerin solution and immersion in phenolic acid in water for one to three months. The body was donated by a person who had given informed consent for use for scientific and educational purposes prior to death.

Two radiologists, specialized in interventional musculoskeletal US, performed eight US-guided injections at four different levels of the sacral spine: the latter was bedded in a prone position on the CT table. All US procedures were carried out with a standard "bedside" US device under default settings (iU22; Philips Ultrasound, Bothell, Washington) with a convex 1-5 MHz curved array transducer. Frequency, depth, focus and gain were adjusted accordingly during the examination. The needle positioning was performed using a sterile transducer cover and a sterile ultrasound gel. Following a CT (Syntec Synergy; GE Healthcare, Vienna, Austria) was carried out to define the respective needle position and the spread of the applied contrast agent. Subsequently the cadaver was dissected: the legs and the upper part of the body were removed, and the pelvis was split into two parts in a median-sagittal orientation. The left half of the pelvis was dissected anatomically, and the right half was used for transverse anatomical cryosections.

\section{Sonographic localization and US-guided pararadicular injection}

To define the respective level of interest for pararadicular injections in the sacral spine the transducer was positioned in a median-transversal orientation to assess the typical transition from the fifth lumbar to the first sacral spinous process as described by Loizides et al [19]. From there the transducer was slightly moved laterally to depict the sacral foramen S1, distinguishable as a discontinuity of the bone (hypoechoic lacuna) between the intermediate 


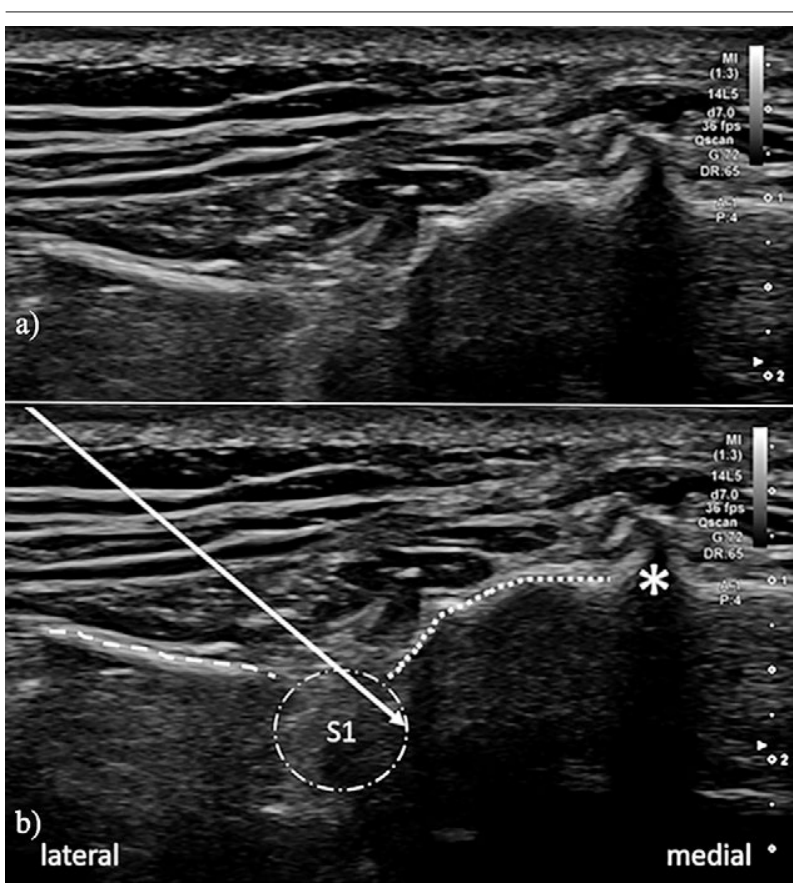

Fig 2. Example of the sono-anatomical access path of the left $\mathrm{S} 1$ nerve root (2a: without annotations, $2 \mathrm{~b}$ : with annotations). For better illustration a linear $14-5 \mathrm{MHz}$ transducer was used. Arrow: intended needle path way, circle: bony boundary of the sacral foramen, well confined by the linear (lateral sacral crest) and dotted (intermediate sacral crest) dash-lines. Star: spinal process of the first sacral bone.

and lateral sacral crest. After defining the sacral foramen of S1, the transducer was moved caudally until the next bone discontinuity was depicted (S2-S4). Once the appropriate level was defined, a spinal needle $(21 \mathrm{~g}, 80 \mathrm{~mm}$; Sterican, Braun, Melsungen, Germany) was introduced from a lateral approach positioned into the respective neuro-foramen. The needle insertion was carried out in an in-plane technique which enables real-time visualization during the whole procedure (positioning and advancement strictly parallel to the long axis of the transducer). The target point was reached when the needle tip showed contact with the inner medial bone surface of the respective sacral foramen (fig 2). Thus, eight needles (one for each sacral foramen on both sides) were positioned. Following $1 \mathrm{~mL}$ of a solution consisting of one-third water-soluble iodinated contrast agent (Iopamidol, Jopamiro $300 \mathrm{mg} \mathrm{J} /$ $\mathrm{mL}$; Bracco, Milan, Italy) and two-thirds of a highly pigmented aqueous blue dispersion (Unisperse Blue B-E; Ciba Specialty Clinicals, London, United Kingdom) was injected into each pararadicular compartment.

The needle tip and the distribution of the injected solution were controlled by means of CT (section thickness: $1.0 \mathrm{~mm}$; pitch: 0.5 ). Then the cadaver, without removing the needles, was transferred to the Division of
Clinical and Functional Anatomy and processed as described below.

\section{Anatomic dissection}

In multiple steps the left half of the cadaver was gradually dissected with the needles left in the placed position to enable clear evidence regarding the correctness of the needle placement. Initially, the skin was skimmed from the underlying adipose tissue and flapped laterally. Then the fatty tissue was removed from the muscle fascia. At the next step, the gluteus maximus muscle was detached from medially until the level of the lateral sacral crest was visible. Subsequently, the posterior sacroiliac and interosseous ligaments were removed so that the posterior sacral foramina could be seen.

The right half of the cadaver was used for transverse anatomical cryosections. For this purpose, this part was deep-frozen and then cut into transversal slices, such as the CT images (through the sacral foramina) with a thickness of $2 \mathrm{~cm}$ to illustrate the injected blue dispersion and its spread along the spinal nerves.

\section{Results}

A CT scan confirmed the correct position of all needle tips within the respective pararadicular compartment of the spinal sacral nerve roots directly adjacent to the inner medial bony boundary of the posterior sacral foramen (fig 3).

The anatomical dissection of the left half of the cadaver enabled once more clear evidence regarding the correctness of all placed needles. Moreover, the dispersion of the blue dye within the dural sac and along the exiting sacral spinal nerves warranted the accurate positioning fig 4).

The transverse cryosections confirmed the accumulation of the blue dye along the dorsal and ventral rami of the spinal nerves (fig 5).

Altogether a $100 \%$ success rate for correct injections could be achieved.

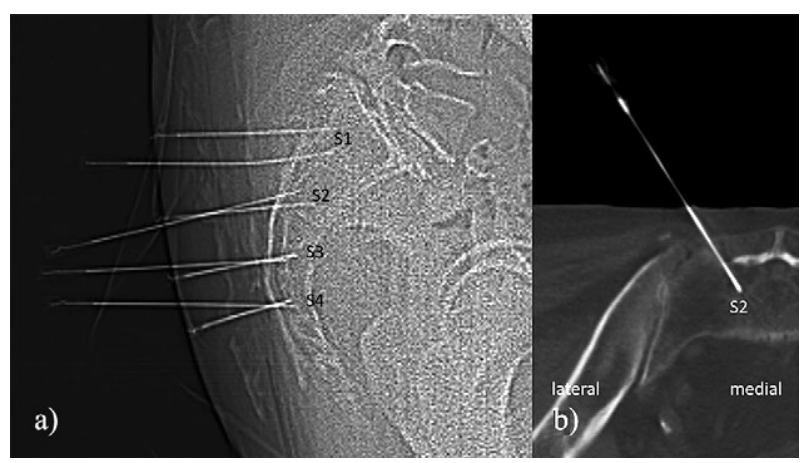

Fig 3. Lateral CT scout with all 8 needles in situ (a). Axial CT scan at the level of the left S2 depicting the correct needle placement within the respective dorsal sacral foramen (b). 


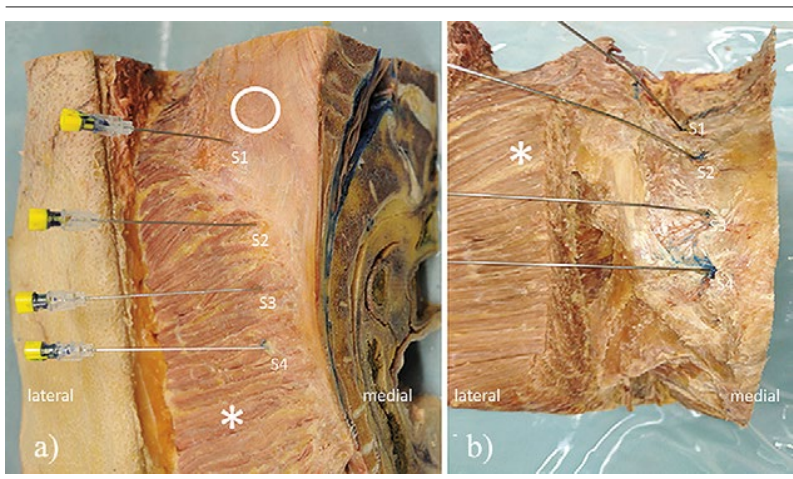

Fig 4. a) Needle placement in the half-split cadaver. In this layer the gluteus maximus muscle is depicted (star); the thoracolumbar fascia is marked with a ring; b) Anatomic dissection of the right semi-pelvis demonstrating the correct needle placement within the four dorsal sacral foramina.

\section{Discussions}

In this study we assessed a new possible approach of US-guided pararadicular injections in the sacral spine and evaluated the preliminary accuracy by means of CT and anatomical dissections.

Lumbosacral radiculopathies are common and depending on the underlying reason and clinical symptoms, different therapeutic options ranging from conservative or symptomatic management to open decompression surgery are available [20]. Based on the fact that slightly more than $90 \%$ of herniated discs occur at the lumbar L4-L5 or the L5-S1 disc space [4], in clinical practice the most frequent references for sacral root injections regard the S1 nerve root. Considering recent literature, imaging guided procedures and therapies have gained in importance and they have been described as a valuable procedure in patients in whom conservative measures are not effective and whenever surgery is not indicated [21]. This may be based on the possibility of better depiction of anatomical structures as well as the evaluation of possible variants. Aguirre et al [22] described in their study a spinal CT-guided infiltration technique for an effective therapy for chronic back pain with a low complication rate, a good response and a high therapeutic value. Also, Fotiadou et al [23] reported spinal injections under CT guidance as being an accurate and safe method that can be used to relieve low back pain and minimize the risk of disability. However, radiation exposure, costs and the needed premises must be considered.

US is in contrast to CT a far cheaper, faster and, moreover, radiation-free method for spinal injections $[11,24]$. Additionally, US is the only imaging modality with the possibility of real time needle guidance and the direct insight to the aiming point [24]. There are many

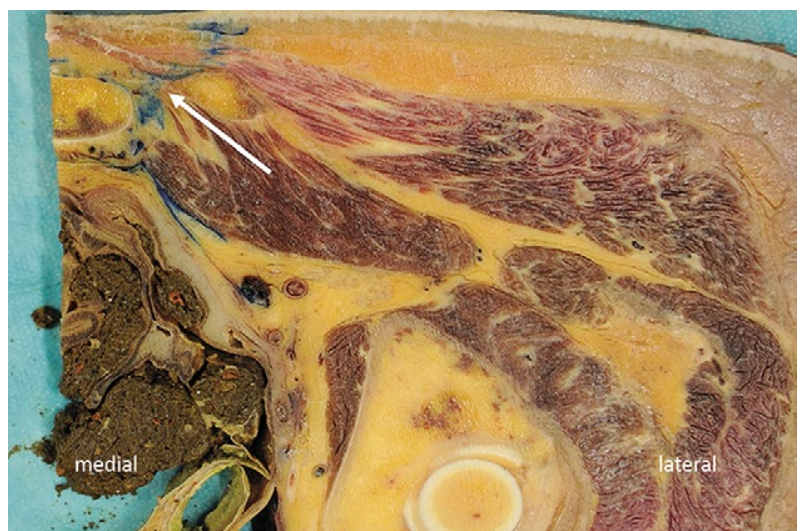

Fig 5. Transverse anatomical cryosection at the level S3 showing the dorsal sacral foramen (arrow) and the accumulation of the blue dye along the dorsal and ventral rami.

published studies that emphasize the usefulness of ultrasound in musculoskeletal and pain medicine $[25,26]$. Concerning injections in the sacral spine only a few studies were performed. Klocke et al [27] were the first to describe a sonographic method for identifying the sacral hiatus landmarks to facilitate real-time guidance of steroid injections into the caudal epidural space. Nikooseresht et al [13] and Kim et al [14] presented in a similar setting that ultrasound can be used as a screening tool for performing caudal epidural injections. Quite recently Kao et al demonstrated in their review article relevant anatomic features and variations of the sacral bone and the development of imaging guided techniques, also including the US-guided approach for performing caudal epidural injections [15], They used a longitudinal transducer position with the sacrococcygeal ligament as the sighted landmark. The presence of unidirectional flow in the color Doppler was defined as a successful caudal epidural injection [16]

To the best of our knowledge this is the first study to evaluate the feasibility and accuracy of US-guided pararadicular sacral nerve injections based on CT correlation and anatomic dissection. Although this is a cadaver study and only a few needles were used, the results have shown that all needle tips were placed correctly. Even though the nerve root cannot be visualized directly, we could show that it is sufficient to position the needle tip at the proposed landmark (inner medial bone surface of the respective dorsal sacral foramen) to gain an adequate accumulation of the injected solution in the pararadicular space. We verified this fact by means of CT scan and anatomical dissections. Further circumstances can be illuminated as following: the dural sack is hung-up and secured via complicated band structures. One of these protections is the anchorage of the outer dural sheath at the sacral 
foramina. In this region, the dura mater spinalis opens toward the exiting nerve roots. Being located between the periost of the spinal channel and the dural sac the epidural space is a virtual cavity and a "true potential space" [28] wherefore it becomes clear, why an injection in this space is sufficient to achieve a pararadicular fluid dispersal [29]. Another point that must be addressed in all USguided procedures is the investigator dependency. A lot of experience and understanding of the sonoanatomy is necessary to perform interventions in a safe and secure as well as accurate manner. However, with anatomical and practical sonographic knowledge interventions are easy to learn. Finally, possible difficulties based on anatomic variants, abnormal deformation of the spine or even hindered conditions due to obesity must be addressed. At this point the correct identification of lumbosacral transitional vertebrae has to be emphasized in order to avoid an intervention or surgery at an incorrect level [30]. In ambiguous cases an X-Ray (Kashoggi et al [31] recommend a antero-posterior Ferguson radiograph, angled cranially at $30^{\circ}$ ) or a CT scan should be performed to clarify anatomical conditions. Nevertheless, US is an excellent tool to perform interventions in the spine, often helping to identify anatomic variants, as for example described by Povo et al [32], to individually assess the patient's situation for gaining optimal results.

Although all the injections were performed on cadaveric models and not on real patients, these preliminary results clearly demonstrate a new and feasible US approach for pararadicular injections in the sacral spine and can be used as a guidance for further research on patients.

In conclusion, although it is known that (sacral) S2 to $\mathrm{S} 4$ radiculopathy is rare, the purpose of this study was not to evaluate the clinical impact, but to assess the feasibility of US-guided injections in the sacral spine. Our new approach for US-guided pararadicular injections of sacral nerves proved to be reliable and accurate in placing the needle in the correct location and can be used as an alternative modality to the existing "ionizing" fluoroscopy and computer tomography. The clinical impact of this new technique will, of course, be further evaluated on an already ongoing prospective randomized clinical trial.

\section{Conflict of interest: none}

\section{References}

1. Alexander CE, Dulebohn SC. Lumbosacral Radiculopathy. StatPearls Publishing. Treasure Island (FL); 2017.

2. Tawa N, Rhoda A, Diener I. Accuracy of magnetic resonance imaging in detecting lumbo-sacral nerve root compromise: a systematic literature review. BMC Musculoskelet Disord 2016;17:386.
3. Tarulli AW, Raynor EM. Lumbosacral radiculopathy. Neurol Clin 2007;25:387-405.

4. Donnally IC, Dulebohn SC. Lumbosacral Disc Injuries. StatPearls Publishing. Treasure Island (FL); 2017.

5. Fish DE, Lee PC, Marcus DB. The S1 "Scotty dog": report of a technique for $\mathrm{S} 1$ transforaminal epidural steroid injection. Arch Phys Med Rehabil 2007;88:1730-1733.

6. Knezevic NN, Mandalia S, Raasch J, Knezevic I, Candido $\mathrm{KD}$. Treatment of chronic low back pain - new approaches on the horizon. J Pain Res 2017;10:1111-1123.

7. Johnson BA, Schellhas KP, Pollei SR. Epidurography and therapeutic epidural injections: technical considerations and experience with 5334 cases. AJNR Am J Neuroradiol 1999;20:697-705.

8. Ozcakar L, Onat SS, Gurcay E, Kara M. Are Blind Injections Ethical or Historical?: Think Twice with Ultrasound. Am J Phys Med Rehabil 2016;95:158-160.

9. Chang A, Pochert S, Romano C, Brook A, Miller T. Safety of 1000 CT-guided steroid injections with air used to localize the epidural space. AJNR Am J Neuroradiol 2011;32:E175-E177.

10. Hurdle MF. Ultrasound-Guided Spinal Procedures for Pain: A Review. Phys Med Rehabil Clin N Am 2016;27:673-686.

11. Loizides A, Obernauer J, Peer S, Bale R, Galiano K, Gruber $\mathrm{H}$. Ultrasound-guided injections in the middle and lower cervical spine. Med Ultrason 2012;14:235-238.

12. Kim YH, Park HJ, Moon DE. Ultrasound-guided Pararadicular Injection in the Lumbar Spine: A Comparative Study of the Paramedian Sagittal and Paramedian Sagittal Oblique Approaches. Pain Pract 2015;15:693-700.

13. Nikooseresht M, Hashemi M, Mohajerani SA, Shahandeh F, Agah M. Ultrasound as a screening tool for performing caudal epidural injections. Iran J Radiol 2014;11:e13262.

14. Kim DH, Park JH, Lee SC. Ultrasonographic Evaluation of Anatomic Variations in the Sacral Hiatus: Implications for Caudal Epidural Injections. Spine (Phila Pa 1976) 2016;41:E759-E763.

15. Kao SC, Lin CS. Caudal Epidural Block: An Updated Review of Anatomy and Techniques. Biomed Res Int 2017;2017:9217145.

16. Park Y, Lee JH, Park KD, Ahn JK, Park J, Jee H. Ultrasound-guided vs. fluoroscopy-guided caudal epidural steroid injection for the treatment of unilateral lower lumbar radicular pain: a prospective, randomized, single-blind clinical study. Am J Phys Med Rehabil 2013;92:575586.

17. Bron JL, van Royen BJ, Wuisman PI. The clinical significance of lumbosacral transitional anomalies. Acta Orthop Belg 2007;73:687-695.

18. Hughes RJ, Saifuddin A. Imaging of lumbosacral transitional vertebrae. Clin Radiol 2004;59:984-991.

19. Loizides A, Peer S, Plaikner M, et al. Ultrasound-guided injections in the lumbar spine. Med Ultrason 2011;13:5458.

20. Mehta N, Salaria M, Salaria AQ. Comparison of fluoroscopic Guided Transforaminal Epidural Injections of Steroid and Local Anaesthetic with Conservative Management 
in Patients with Chronic Lumbar Radiculopathies. Anesth Essays Res 2017;11:17-22.

21. Uhlenbrock D, Arlinghaus J. Results of CT-guided periradicular pain therapy. Rofo 1997;166:528-534.

22. Aguirre DA, Bermudez S, Diaz OM. Spinal CT-guided interventional procedures for management of chronic back pain. J Vasc Interv Radiol 2005;16:689-697.

23. Fotiadou A, Wojcik A, Shaju A. Management of low back pain with facet joint injections and nerve root blocks under computed tomography guidance. A prospective study. Skeletal Radiol 2012;41:1081-1085.

24. Loizides A, Gruber H, Peer S, Galiano K, Bale R, Obernauer J. Ultrasound guided versus CT-controlled pararadicular injections in the lumbar spine: a prospective randomized clinical trial. AJNR Am J Neuroradiol 2013;34:466-470.

25. Chang KV, Wu WT, Huang KC, Jan WH, Han DS. Limb muscle quality and quantity in elderly adults with dynapenia but not sarcopenia: An ultrasound imaging study. Exp Gerontol 2018;108:54-61.

26. Chang KV, Wu WT, Han DS, Ozcakar L. Static and Dynamic Shoulder Imaging to Predict Initial Effective- ness and Recurrence After Ultrasound-Guided Subacromial Corticosteroid Injections. Arch Phys Med Rehabil 2017;98:1984-1994.

27. Klocke R, Jenkinson T, Glew D. Sonographically guided caudal epidural steroid injections. J Ultrasound Med 2003;22:1229-1232.

28. Newell RL. The spinal epidural space. Clin Anat 1999; 12:375-379.

29. Hormann R, Brenner E, Kunzel KH. Funktionelle Aspekte der Dura sacralis und des anokokzygealen „Verspannungsapparates". Osteopathische Medizin; 2016;17:4-8.

30. Konin GP, Walz DM. Lumbosacral transitional vertebrae: classification, imaging findings, and clinical relevance. AJNR Am J Neuroradiol 2010;31:1778-1786.

31. Khashoggi KG, Hafiz RM, Bock YM, Kaki AM. Determination of lumbosacral transitional vertebrae in kidney urinary bladder $\mathrm{x}$-ray films in the Saudi population. Saudi Med J 2017;38:794-797.

32. Povo A, Arantes M, Matzel KE, et al. Surface anatomical landmarks for the location of posterior sacral foramina in sacral nerve stimulation. Tech Coloproctol 2016;20:859-864. 